\title{
Medical Society for the Study of Venereal Disease Seventy Fifth Spring Meeting: Oxford 1997
}

MSSVD

\author{
Andrew Winter, Jonathan Ross
}

Keble College, Oxford hosted the 75th spring meeting of the MSSVD from 3 to 6 July 1997. A chill reminder of past global catastrophe greeted the 320 delegates on the first morning - a huge dinosaur skeleton which dominates the concourse of the university museum where the main lectures were held. This set the scene for a meeting with a refreshingly global perspective. Themes of epidemiology, disease control, and the catastrophe of untreated or even untreatable infections ran through most of the 10 keynote addresses which we review here. In addition, 31 oral presentations were given and 62 posters presented.

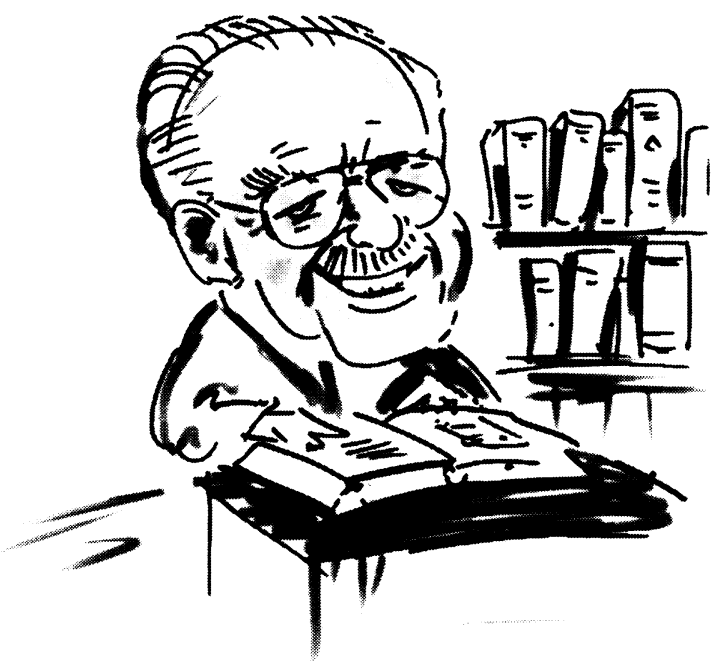

Dr $R$ Morton reviewed the 75 year history of the MSSVD and celebrated his own 80th birthday during the Oxford spring meeting. Acknowledgement: Doug Ayre, Caricature Art, 3M Health Care.

\section{Epidemiology, disease control, and prevention}

RATIONING

Professor Sir Leslie Turnberg, president of the Royal College of Physicians, opened the conference with a call for doctors to take an active part in the debate about rationing. Covert rationing as practised now was unfair, unjust, and discriminatory, he claimed. The college desired open debate and public discussion to help set priorities for health care.

\section{DISEASE BURDENS AROUND THE WORLD}

A series of presentations graphically illustrated the huge burden of sexually transmitted infections (STIs) around the world. An estimated 333 million new STIs were diagnosed in 1995 worldwide, said Professor Michael Adler of University College, London.
Globally, STIs are the second commonest cause of women losing healthy life. Professor $\vec{\Rightarrow}$ King $K$ Holmes of the University of $\frac{0}{0}$ Washington, Seattle explained that in the United States the estimated cost of STIs and their sequelae (including pelvic inflammatory $\mathbb{D}$ disease) was over US $\$ 7$ billion in 1994, exceeding the total costs of HIV infection. The incidence of syphilis in eastern Europe and the Russian Federation $\vec{\omega}$ is rocketing, according to data prepared by Professor Gromyko of the WHO, Copenhagen, and presented by Dr Adrian w Renton of the Centre for Research on Drugs or and Health Behaviour, London. Within devel- $\vec{\infty}$ oped countries, extremely high rates of STIs in deprived urban areas demonstrate our failure adequately to deliver effective health care. $\bigcirc$ Professor $\mathcal{F}$ Zenilman from Baltimore presented compelling evidence for massive differences in $\mathbb{\Phi}$ STI incidence between different ethnic $\vec{\bullet}$ groups. The overall incidence of gonorrhoea in Baltimore City was 1199 per 100000 in 1995. Within the core group area, which comprises over $99 \%$ African Americans, the rate among those aged $15-39$ was 8100 per 100000 in men and 4900 per 100000 in $\mathbb{D}$ women, rising to $12000-15000$ per $100000 \stackrel{\Rightarrow}{\Rightarrow}$ for adolescents. He reminded the audience $\frac{9}{3}$ that there was nothing new in this-back in the 1940s non-white American conscripts had five times the incidence of syphilis as their of white colleagues. So why do non-white Americans suffer more sexual infections than white Americans? Evidence for different bio- $\delta$ logical risk for STIs between ethnic groups is weak, and access to sexual disease services is 욱 reasonably equitable. There is also surpris- $\rightarrow$ ingly little evidence of gross differences in sexual behaviour between ethnic groups. $\tilde{N}$ According to the 1988 American National i Survey of Family Growth white 15 to 19 year N old women were just as likely to have ever had 0 sexual intercourse as black women $(51 \%$ and

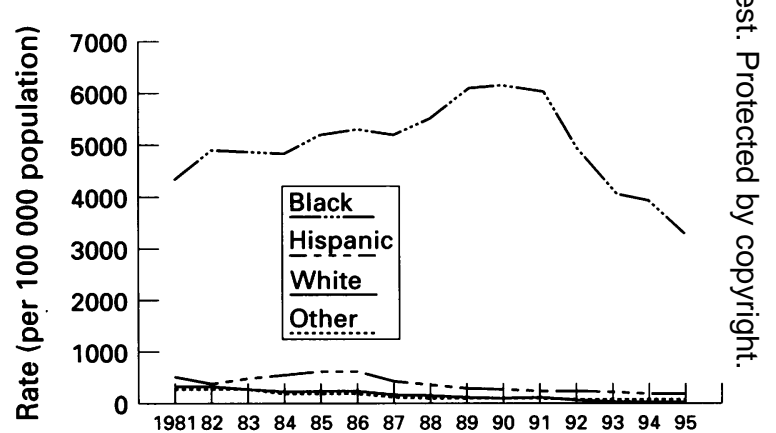

Gonorrhoea-reported rates for 15-19 year old males by race and ethnicity: United States, 1981-95. 
$59 \%$ respectively). Indeed, in the 1991 Center for Disease Control Youth Risk Behavior Report more black teenagers than white reported using condoms at last intercourse. In the United States and probably elsewhere a complex interplay between socioeconomic status, unemployment, drug use, migration, and subtle differences in sexual partner networks explain the link between non-white ethnicity and high STI incidence. While the United States has linked disease notification to ethnic status for years (and not always for the most honourable reasons), Professor Kevin de Cock of the London School of Hygiene and Tropical Medicine lamented the failure of the United Kingdom to do this. Picking his way carefully through an ethical and political minefield, he referred to the covert rise in the incidence of tuberculosis among people of African origin within London, with an incidence of 135 per 100000 in 1993, some 30fold higher than people of non-African origin. Linking ethnic origin with disease notification would have allowed earlier recognition of this epidemic. Ethnicity should be regarded as a "sociological" rather than a racial or biological marker, Dr Renton commented during the discussion. There seemed no point in recording ethnic data without having a clear plan about how and where to intervene with preventive strategies. We should strive further to identify the subpopulations most at risk.

The global HIV epidemic has in turn spawned an epidemic of tuberculosis. Of some eight million cases occurring annually worldwide, three million are never treated, and only half a million receive WHO recommended directly observed therapy, according to Dr Anton Pozniak, senior lecturer at King's College Hospital, London. Resistance to both rifampicin and isoniazid defines multidrug resistant tuberculosis (MDR-TB). MDR-TB has been created entirely by improper use of precious anti-TB drugs in poorly implemented and poorly managed TB control programmes. In fact, only 48 of 214 countries have anything like effective tuberculosis control programmes. MDR-TB rates in the United Kingdom remain low, about $1.4 \%$ in 1994. Among Haitian immigrants to the United States, however, $32 \%$ of TB isolates had primary rifampicin resistance. Recent infection accounts for $23 \%$ of all TB in London, and up to $70 \%$ of TB in recent outbreaks of MDR-TB in New York. Molecular analysis to detect drug resistance is still in its infancy, although the rpoB line probe assay for rifampicin resistance appears promising.

\section{THE FIGHT AGAINST SEXUALLY TRANSMITTED} INFECTIONS

So what can be done to reduce the global burden of STIs? Sweden is renowned for its efforts in controlling genital Chlamydia trachomatis infection. With the help of just a little maths, Professor T Ripa of Lund University explained how to contain a sexually transmitted infection in a community. The reproductive rate of an infection is a function of the following:
- transmission efficiency

- duration of infection

- mean rate of partner change

- the variance of the mean rate of partner change (reflecting core group behaviour).

These factors can be influenced by condom use, screening, case finding and treatment, sex education, and promotion of health seeking behaviour. The Halmstad programme described by Professor Ripa saw genital $C$ trachomatis incidence fall from 9000 per 100000 to 1500 per 100000 over 10 years among 20-24 year old women. He believed their success was due to (i) a policy of universal screening for all sexually active women and symptomatic men less than 30 years old, (ii) proper laboratory support, and (iii) frank sexual disease education which created a climate of "sexual health seeking behaviour".

Diagnostic tests for genital $C$ trachomatis infection were reviewed by Dr Angelika Stary of the University of Vienna. A host of molecular amplification methods are now available or undergoing evaluation. In symptomatic patients, the test performance of culture, ELISA, immunofluorescence, and molecular amplification is probably equivalent. The key advantage of the new molecular methods is their ability to detect asymptomatic infection with less invasive specimens. Use of such techniques has helped uncover a silent epidemic of chlamydia. Although urine testing seems attractive, inhibitors against the ligase chain reaction (LCR) and transcription mediated amplification (TMA) can be a problem. Vulval swabs are not so affected and are also easier to process than urine.

Professor King $K$ Holmes presented convincing endometrial biopsy data confirming the role of anaerobes in the pathogenesis of pelvic inflammatory disease (PID). Treatment for PID should include as a minimum agents active against Neisseria gonorrhoea, $C$ trachomatis, and anaerobes, he believed, although there are as yet no randomised controlled trial data to support such anaerobic cover. The global burden of PID is incalculable and is exacerbated by coinfection with HIV which increases the risk of developing tubo-ovarian abscess threefold. Few women with symptoms of PID attend for medical help, and those that do generally receive inadequate care. In Lima, Peru, medical students attended local pharmacies pretending to be women with symptomatic PID. Almost $90 \%$ were offered some medication but none at all were offered correct treatment. Local doctors did little better, with only $30 \%$ offering an antichlamydial antibiotic and none treating anaerobic infection. Professor Holmes was too diplomatic to cite work showing that women with PID sometimes fare little better in primary care units and emergency departments in the United Kingdom. Prevention of PID is far better than cure, and he cited an intervention study set in a managed care programme in the United States where screening for genital $C$ trachomatis more than halved the prevalence of PID.

One clear example of successful sexual health screening is the cervical cytology pro- 
gramme, which reached about $85 \%$ of its target population in 1995, according to Professor $\mathcal{F}$ Cuzick, of the Imperial Cancer Research Fund, United Kingdom. Cervical cancer incidence is now falling by about $8 \%$ per year in England and Wales. The case for screening for high risk human papilloma virus (HPV) as an adjunct to cytology is strengthening. In a study of 2000 routine smears taken in a family planning clinic, HPV testing doubled the detection of high grade CIN III lesions. Problems remain translating this technology into a failsafe screening tool since cytology still detects lesions missed by HPV testing.

Professor Michael Adler reminded us that the vast majority of those with STIs never get past the front door of healthcare facilities, as they are asymptomatic, or not inclined to seek (or able to afford) medical help. In the conceptual framework for STI control developed in conjunction with the United Kingdom Overseas Development Administration, the benefits of mass treatment in areas of high STI prevalence were explored and we await the results of trials of this strategy in Uganda. In a moving talk, $\mathrm{Dr}$ Michael Waugh, president of the International Union against Sexually Transmitted Infections (previously the IUVDT), called for more institutions and individuals within the United Kingdom to join the fight for better sexual health worldwide, bringing knowledge and skills to bear with tact and sensitivity.

\section{HIV infection}

VIRAL DYNAMICS

The Society was greatly honoured that $\mathrm{Dr}$ David $\mathrm{Ho}$, director of the Aaron Diamond AIDS Research Foundation, New York, agreed to give the inaugural Duncan Catterall Memorial Lecture. Developing a familiar theme of HIV viral dynamics, Dr Ho presented further data from his cohort of 20 acute seroconvertors who have completed at least 17 months of therapy with highly active triple combination therapy in an attempt to prove the concept of viral eradication. An extensive hunt for infectious virus or viral RNA in samples of blood, cerebrospinal fluid, seminal plasma, tonsil, or cervical lymph node has proved fruitless in those fully complying with therapy. Unspliced viral RNA and, less frequently, proviral DNA have been detected in gut associated lymphoid tissue in some patients but so far there has been no sign of viral replication. He stressed that the mathematical models used to predict "burnout" of virus were firmly based on real patient data. The model has been refined slightly to give an estimated half life for HIV viral turnover of $1 \cdot 1$ days in productively infected $\mathrm{T}$ lymphocytes, 8.5 days in lymphocytes, and 14.4 days in the macrophage compartment. Recent estimates based on a macaque SIV model suggest that the half life of extracellular virus is under 10 minutes, requiring production of up to $10^{11}$ virus particles per day. Dr Ho emphasised that ultimately successful combination antiretroviral therapy must force the virus into a reproductive corner, where four to five highly disadvantageous mutations are required for virus survival.

MORE ON THE DELTA TRIAL

Professor Fonathan Weber of Imperial College, London, presented preliminary data from the extended virology study of the DELTA trial. Originally, the virology subset of the study consisted of 242 patients. Now HIV quantita- $\frac{\bar{\sigma}}{\bar{N}}$ tive viral loads are available for 1280 subjects, measured by the NASBA assay on stored $\triangle$ serum. The results in general confirm earlier is findings which support the predictive power $\vec{\circ}$ and importance of viral load testing. Three other points emerged:

- the nadir viral load after initiating antiretrovirals is an important predictor of $\omega$ future treatment failure. Those who or failed to suppress virus load below 10000 copies $/ \mathrm{ml}$ at week 16 had over six $\infty$ times the mortality of those who responded completely $(<800$ copies/ $\mathrm{ml}$ );

- those with lower CD4 counts failed therapy earlier, which might support earlier initiation of treatment;

- when adding a single drug to an existing regimen (for example, zidovudine), complete suppression $(<800$ copies $/ \mathrm{ml}$ ) was observed in only $60 \%$ of those with low viral loads $(<20000$ copies $/ \mathrm{ml})$ and just $10 \%$ of those with a viral load over 150000 copies $/ \mathrm{ml}$. This supports the current trend towards changing two or more drugs of a failing combination.

\section{Other highlights}

A sparkling lecture from $\operatorname{Dr} \mathcal{F} W$ Maunder, a medical entomologist at Cambridge University, enlisted our sympathy for the female Sarcoptes scabeii mite, and corrected many historical errors in textbooks. Oxford consultant dermatologist $\mathrm{Dr}$ Wojnarowksa shared her extensive experience in managing lichen sclerosus. Her current protocol for 0 biopsy confirmed cases includes screening for $\omega$ other associated autoimmune disease and then treating with high potency topical corticosteroid for at least 3 months in an attempt to avoid scarring. Indefinite follow up is mandatory because of the $5-10 \%$ risk of malignant transformation. Dr $S$ Ralph of Leeds General Infirmary won both prizes, for the best oral $\stackrel{\mathbb{D}}{\circ}$ and best poster presentation, for work describ- 2 ing the effect of bacterial vaginosis on early trimester miscarriage and an audit of conservative management of CIN 1 . 